\title{
Theory of special relativity: False premise that leads to obtain incorrect conclusions
}

\author{
Enrique Martinez Viladesau \\ Escuela Técnica Superior de Ingenieros Industriales de Barcelona (ETSIUB), Spain \\ E-mail addresses: emviladesau@gmail.com
}

Received March 2018

Received in revised: March 2018

Published : June 2018

\begin{abstract}
In this paper, we present the chain of errors that have been committed to accept the utopia of time dilation. This chain of errors begins accepting as true some false premises. Starting from these false premises, errors of interpretation of reality are triggered, which lead to incorrect conclusions.
\end{abstract}

Keywords: Relativity, Time dilatation

C2018 The Authors. Published by Fundamental Journals. This is an open access article under the CC BY-NC https://creativecommons.org/licenses/by-nc/4.0/

https://doi.org/10.14331/ijfps.2018.330117

\section{INTRODUCTION}

Time dilation, in the theory of special relativity is the slowing down of a clock as determined by an observer who is in relative motion with respect to that clock. It is also defined a difference of elapsed time between two events as measured by observers. But here we arrive at the fallacy of the time dilatation, from an incorrect idea. Albert Einstein (Einstein, 1948) in his book, mentioned about a train that moves with rectilinear and uniform speed with respect to the embankment that serves as support for its roads. In this paragraph he says that, the time needed for a process in relation to the car cannot be equated to the duration of the same process judged from the reference body of the embankment. When we take this statement as a premise, we see that it is the beginning that leads us to a false conclusion. In order to unmask the error that this statement contains, we must specify the meaning of the word process that it uses in its affirmation. Here we consider as process the occurrence of two or more events linked to each other and that occur in the outer space keeping a certain sequence in its execution and that requires a certain execution time. We can consider that it is a physical, chemical or biological process. Thus, when mentioning, the time a process needs, this time is inherent to this process and will serve to identify it. We will call it, own time $t_{p}$ of the process. Another issue is the observation time $t_{o}$ that requires seeing this process from different Situations of outer space. 


\section{THE MENTAL EXPERIMENT OF THE TRAIN VAGON}

To visualize the statement cited in the aforementioned book, (Einstein, 1948), some physical treatises expose the mental experiment of the train car or the mirror by means of Figure 1.

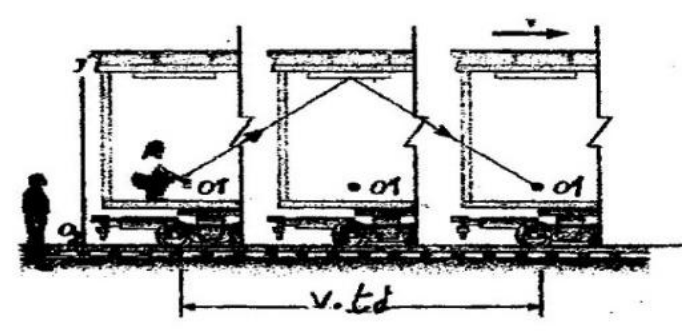

Fig 1

The drawing represents some cuts of the train car, in different situations due to its rectilinear displacement and at constant speed $v$. An observer $O_{1}$, who is mounted on the train car, fires a ray of light, which is reflected in a mirror located on the roof of the same. The observer is fixed on the ground and is located just at the beginning where the ray is triggered. In this case the process consists of the round trip of a ray of light thrown by the operator $O_{1}$, from the base of the train car. In this mental experiment we try to justify the time difference that exists between the two observers when measuring the duration of the light beam.

\section{ERROR COMMITTED BY ACCEPTING THE QUOTED PREMISE}

In the premise that we had previously commented, the error is the following: Consider as if there were two distinct own times for the same process when in reality it is two observation times of a single process from two different observation points; that of the operator $O_{1}$ and that of the observer who is fixed on the ground. Figure 2 represents this situation.

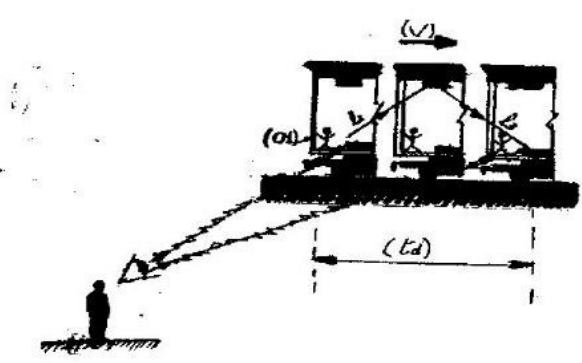

Fig 2

Considering as observation time $t_{0}$ the period of time from when the beam is triggered until it returns to the base of the train carriage. The observation time $t_{0}$ of the operator $O_{1}$, which is mounted on the train car, is in fact the same as the own time $t_{p}$ of the process. The observation time, $t_{0}$ of an observer located fixed in any point of the ground, could never be assimilated to the own time $t_{p}$ of the process, not even in the case of placing this observer just at the point where it starts. The shot of the ray of light. We believe that the confusion can come from trying to consider as a single physical phenomenon the intervention of two different physical phenomena: the reflection of a ray of light and the movement of the platform that translates to the mirror. Although we must consider that they are simultaneous, we will comment later on the event of the simultaneity.

\section{THE VITAL TIME OF NONE OF THE TWO TWIN BROTHERS HAS NOT BEEN EXTENDED}

Recall the paradox of the twin brothers that is told in some books, in which one of the brothers is a cosmonaut who undertakes a journey in outer space at a speed approximately to that of light while the other twin brother is It remains on planet Earth. When the astronaut brother returns from his trip you can see a great difference in age between the two brothers (Fig 3).

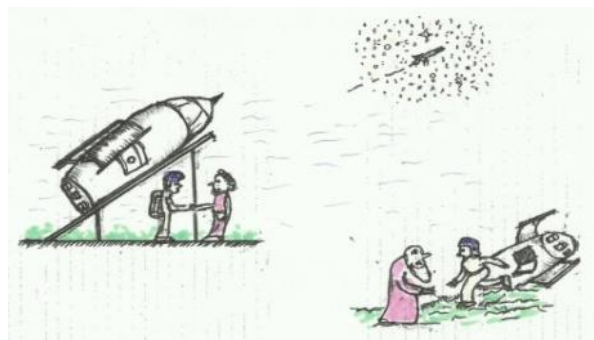

Fig 3

Regarding this assessment, which continues to be more than a science fiction story, we must comment on the following. From what we have explained in the previous paragraphs we can say that: The observation time is extended. We cannot say that the own time of the process is extended. Figure 4 represents the train car traveling at a constant and rectilinear speed $v$. Inside there is a pendulum clock and the process that an observer will register inside the car will be the (Tic-Tac) of the pendulum.

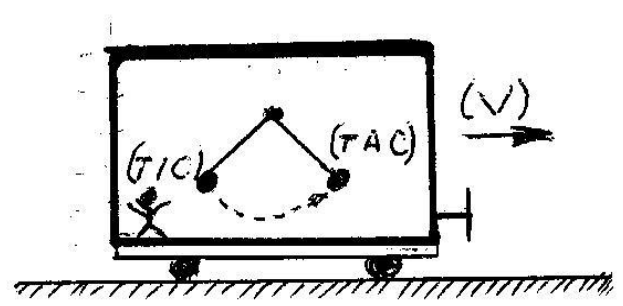

Fig 4

The time interval in which this occurs (Tic-Tac) will measure the passage of time. In this way the observer inside the car can measure the time that is passing. We can admit that the observation time is enlarged with respect to an observer (SRF) that is fixed on the ground. Figure 5 tries to represent this situation:

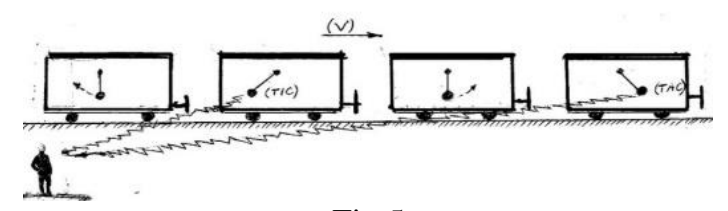

Fig 5 
The fact that the time of observation is enlarged does not mean that time is dilated and justifies the utopia of the example of the twin brothers. The twin brother who stayed on land, if through a hypothetical telescope could see the referred pendulum, would have larger intervals of oscillation, but this would not justify that the real time was not the same for the two brothers

\section{CONTRADICTORY CONCLUSIONS THAT WE OBSERVE IN THE DECLARATIONS OF A. EINSTEIN}

The question that we can surely ask is that, What do we consider that A. Einstein intended to justify in his theory of relativity? Taken from the book by A. Einstein (Einstein, 1948) page 18, announces as, First Principle of Relativity; If one (SRM) moves with respect to one (SRF) then the natural phenomena proceed with respect to the (SRM) according to identical general laws with respect to the (SRF). Physical phenomena occur in the same way. The same physical variables that intervene must be considered and their mathematical formulas of calculation will be the same. It is assumed that we already know the concepts of relative movements and that is understood by inertial reference systems, so we are able to understand the answer to the question: what do we consider that A. Einstein intended to justify in his theory of relativity? The answer is also found in his book (Einstein, 1955) (Page 38). There, the author notes the validity of the Inertial Reference Systems (IRS) to be able to examine and measure the laws of nature in the same way and with the same results in each one of them. Figure 6 is intended to represent a case in which this Principle is fulfilled. Suppose a train car (SRM), which moves on the track in a straight line and with a constant speed $v$ with respect to a person who is still on the ground (SRF).

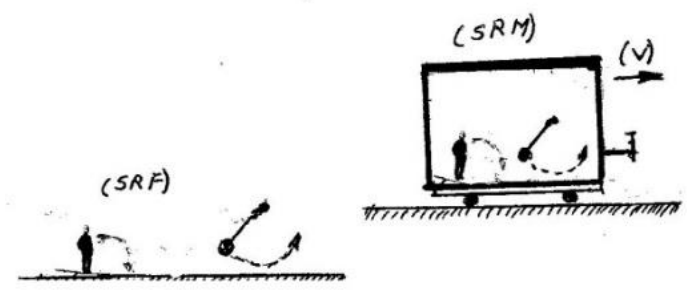

Fig 6

The figure represents the fact that in the (SRM) physical phenomena, parabolic shooting and oscillation of a pendulum, can occur in the same way they occur in the (SRF). The same variables act and comply with the same calculation formulas. In our book (Enrique Martínez Viladesau, 2015), we comment Galileo's experiment of the ship that contains in his hold, birds, flies and drops of water are also mentioned. Besides, the concepts of inertial reference systems and that we understand by position and situation.

According to the opinion that we have been able to draw from the readings of his books, perhaps somewhat disconnected, we believe that one of the three wishes of A. Einstein was that the First Principle of Relativity, that we have described be fulfilled. We will discuss the other two wishes on another occasion. We see that the first principle of relativity is contradicted by the premise in which the physicist departs to consider two different processes.

\section{FALSE PREMISE IN THE EXHIBITION OF THE CONCEPT OF THE RELATIVITY OF SIMULTANEITY}

In the aforementioned book by A. Einstein, in paragraph 9, page 27, and with the title: "The relativity of simultaneity", it says " two events, for example two beams, that are simultaneous with respect to the embankment, are not with respect to the train and vice versa. Each reference body (coordinate systems) has its special time. A temporary location only makes sense when the reference body to which it refers is indicated". As we will see, this statement can also lead us to accept the fallacy of dilation of time. This statement will be identified as: second premise. We highlight the difference that exists with respect to the Premise that we have commented on in previous paragraphs. Ahota we are talking about events not processes, so it will be logical to take care of the observation of the vision of the event and not of the observation of the duration. That is, we do not consider, or do not know, own time. However, it seems that the well-known Physicist wants to lead us to consider two different phenomena. Figure 7 will serve as a support to justify what we are saying. In it we represent the two rays that have been generated between the clouds. It is evident that we are not interested in the process corresponding to the atmospheric phenomenon that has produced them. We represent the train car (SRM) on which an observer moves, which, in this case, is not the one that generates the ray of light. This observer, according to the second premise, agrees to see each of the two rays in two different positions of their displacement,

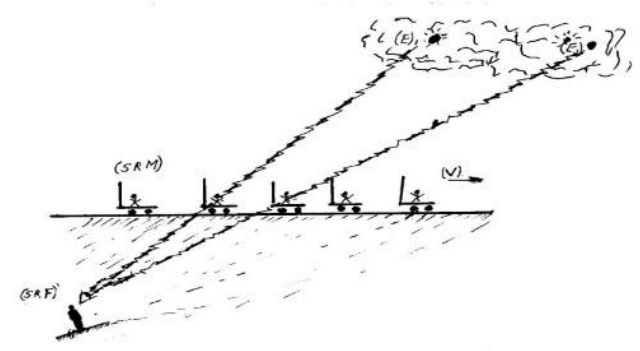

Fig 7

In this difference of observation and, consequently, in different moments of time, we could fall into the error of admitting the fallacy of time dilation. That is to say, to think that two rays whose difference of appearance is zero, and that this will be observed by the observer that is fixed on the ground (SRF), the mobile observer sees them in two different times. So if in the First Premise it was intended to justify the lengthening of the processes. in this Second Premise it was intended to justify the lengthening of the vision of the events. We believe that in the case of events the error that is committed when accepting the Second Premise is double. On the one hand, the error is made of pretending to draw results from the combination of two different physical phenomena: the vision of two rays and the displacement of the train car. On the other hand we must consider as one single event the simultaneous appearance of two or more events. What deserves our attention and classifies the event is simultaneity. It is not the type of event that occurs, since we could admit this characteristic as selection criteria of several, simultaneous and different Events among themselves. To finish this topic, we must add the following: We have exposed the criticism of this second premises to try to 
demonstrate the error of concept that is part. However, we could have already stated that the mental experiment proposed by the author is a chimera. We ask ourselves: at what speed $v$ should the car have to circulate so that the observer who is riding on it could see the two rays sequentially? We believe that it should be superior to the speed of light $c$.

\section{FALSE PREMISE IN THE EXPOSITION OF THE DELIVERY IN THE BAR OF MEASURING}

So far we have talked about the lengthening of time. Now we have to talk about another concept that is even more contradictory and twisted. It is about the longitudinal elongations. This fallacy indirectly implies the mass of a body. In paragraph 10 of the aforementioned book by A. Einstein (Einstein, 1948) speaks of a measuring rod distances. This bar is used both above the car and in the way to measure the same distance, examining the ends of it. The conclusion that comes with these two measures is: if the man in the car travels in a unit of time the distance w measured from the train, this stretch measured from the track, does NOT have to be equal to w. What it claims to affirm is that the lengths of the bodies located in an (SRM) when being observed from a (SRF) have been dilated. This is another principle that leads us to wrong conclusions. We believe that it should say; I would not have to verse from a (SRF) with the same amplitude of measurement. Figure 8 tries to highlight the error contained in the aforementioned premise

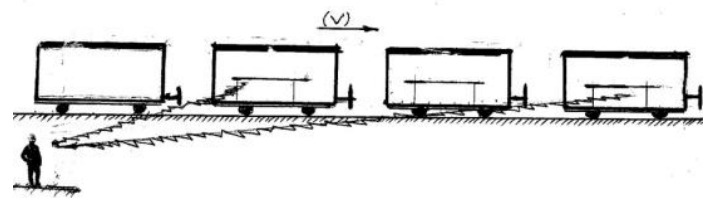

Fig 8

\section{REFERENCES}

Einstein, A. (1948). The Special and General Theory: Prabhat Prakashan.

Einstein, A. (1955). The Meaning of Relativity. Including the Relativistic Theory of the Non-symmetric Field, by Albert Einstein: NJ, Princeton University Press.
Here we will apply the same reasoning that we have used in the previous two false Principles. The actual length is what the observer measures above the car. We can call it your own length. The other length is what the observer sees and we can call it observation length. This wrong principle raises doubts when it comes to raising the famous formula $E=m v^{2}$. Doubt we have seen it appear when we show how to obtain this formula, at the moment of incorporating the linear momentum $P$, which we know is expressed as: $P=m v$, where $m$ is the mass and $v$ speed. At this time we have seen that the mass is corrected by applying the Lorentz Factor $L$. That is to say $P=$ $L m v$. But, what is more curious, at the end of the development is mysteriously dispensed with the existence of this Factor, giving the formula as we know it. This way of acting may demonstrate the way of not knowing how to get out of a situation in which something is left over in the approach to obtaining the formula what we have left is to accept the third premises that we are discussing in this paragraph. Criticism about the validity of the fallacy of dilation of time and dilated of mass, we have divided into two parts. A part we have exposed in the present Essay. As we have seen, we have tried to highlight the error that is committed by accepting the aforementioned Premises as valid and arriving with it to obtain wrong conclusions. Another part of our research is dedicated to demonstrate how it has been tried to validate the answers obtained using mathematical calculations and physical devices (Enrique Martinez Viladesau, 2018). In it we observe the error that is made when trying to compare the time of the process with the time of observation, as if it were of the same nature, proposing an equality instead of an equivalence. It also shows where lies the error of interpretation of the reading of atomic clocks; communication systems (GPS).

Viladesau, E. M. (2015). Teoría de la relatividad. Una falsa teoría: Punto Rojo Libros.

Viladesau, E. M. (2018). Theory of relativity-atomic watches and time dilation. International Journal of Fundamental Physical Sciences, 8(1), 1-4. 